\title{
Les randonnées philosophiques de David Abram et l'argument pour une fondation philosophique de l'animisme
}

David Abram's Philosophical Hiking and the Argument for the Philosophical Foundation of Animism

\section{Stefan Kristensen}

\section{OpenEdition Journals}

Édition électronique

URL : https://journals.openedition.org/cps/4783

DOI : $10.4000 /$ cps. 4783

ISSN : 2648-6334

\section{Éditeur}

Presses universitaires de Strasbourg

\section{Édition imprimée}

Date de publication : 30 mai 2021

Pagination : 141-155

ISBN : 979-10-344-0088-1

ISSN : $1254-5740$

\section{Référence électronique}

Stefan Kristensen, «Les randonnées philosophiques de David Abram et l'argument pour une fondation philosophique de l'animisme », Les Cahiers philosophiques de Strasbourg [En ligne], 49 | 2021, mis en ligne le 30 mai 2021, consulté le 08 novembre 2022. URL : http://journals.openedition.org/cps/4783 DOI : https://doi.org/10.4000/cps.4783

\section{(c) (7) (2)}

Creative Commons - Attribution - Pas d'Utilisation Commerciale - Partage dans les Mêmes Conditions 4.0 International - CC BY-NC-SA 4.0

https://creativecommons.org/licenses/by-nc-sa/4.0/ 


\title{
Les randonnées philosophiques de David Abram et l'argument pour une fondation philosophique de l'animisme
}

\author{
Stefan Kristensen
}

David Abram est un philosophe, randonneur, prestigiditateur et conférencier états-unien dont l'œuvre permet d'entrevoir, et même de sentir clairement, en quoi pourrait consister une philosophie animiste contemporaine. Abram s'inscrit dans le courant de la philosophie environnementale ou d'écologie profonde à la suite d'Arne Næss, mais, à la différence de Næss, il adopte explicitement une perspective phénoménologique qui s'attache à décrire l'expérience vécue de notre subjectivité corporelle dans la résonance avec le paysage et la terre. L'un des intérêts de lire son ouvre est de tirer les conséquences éthiques, intimes, mais aussi politiques de ces interrelations sensibles. La phénoménologie animiste d'Abram n'est pas seulement une sagesse individuelle, une éducation de la capacité de sentir de chacun à l'époque de la crise environnementale; c'est aussi un fondement puissant pour la réflexion et l'action politique. "Devenir animal», restituer la part animale de la subjectivité humaine, est une nécessité afin de faire l'expérience et poser les fondations d'une société qui s'intègre pleinement dans les cycles naturels et qui ne les épuise plus. L'objet des pages qui suivent est cependant plus modeste: on cherchera ici à décrire la manière singulière dont David Abram articule l'héritage philosophique et l'expérience concrète de la nature. Sur cette base, je discute brièvement des fondements métaphysiques d'un animisme contemporain, et je suggère ce faisant une stratégie pour récupérer notre "indigénité» d'Occidentaux. 


\section{Le «devenir animal» du sujet humain}

Pour effacer d'emblée un possible malentendu qui pourrait naître en prenant connaissance du titre de ce livre, David Abram n'a pas écrit un livre deleuzien. Son "devenir animal» a certes des affinités avec celui de Deleuze et de Guattari dans leur ouvrage Mille plateanx, mais deux différences fondamentales doivent être soulignées: premièrement, il s'agit pour Abram d'interroger le sens de l'existence humaine à travers la dimension animale de notre expérience. Il s'agit, comme il l'écrit dans une longue note en bas de page de l'introduction, de «devenir plus profondément humain en reconnaissant, en affirmant et en assumant notre animalité $»^{1}$, et non pas de dissoudre ou de réduire la subjectivité humaine. En quelque sorte, le devenir-animal chez Guattari et Deleuze est un processus qui s'opère contre la maîtrise du sujet humain, comme une contagion qui se propage et installe un régime de vie différent chez les humains: "Nous disons seulement que les animaux sont des meutes et que les meutes se forment, se développent et se transforment par contagion $"^{2}$. Devenir animal dans le propos de David Abram est au contraire un approfondissement de l'expérience humaine, c'est l'humain comme animal dont il s'agit. Il n'y a aucune prétention à se laisser habiter par une autre identité d'animal ou par une meute ou un essaim - c'est d'ailleurs pourquoi il écrit l'expression sans trait d'union, contrairement à Deleuze et Guattari.

Deuxièmement, Abram veut se distancier de l'appétit métaphysique de Deleuze; il est «bien trop pris dans l'expérience vécue - dans la rencontre ressentie entre notre corps de sensations et la terre animée» pour que la "production de concepts abstraits" de Deleuze puisse lui convenir. Ce rapide prologue concernant les relations avec Deleuze, cette mise au point sommaire qu'il fait au détour d'une note en bas de page, permet de situer sa perspective clairement. Différemment du métaphysicien Deleuze, Abram revendique une pratique phénoménologique, et différemment du penseur spéculatif Deleuze, Abram ne cherche pas à s'affranchir de l'expérience humaine - seulement (si l'on peut dire) à l'approfondir

1 D. Abram, Becoming Animal. An Earthly Cosmology, p. 10. La traduction française en cours est à paraître aux Éditions Dehors, par les soins du soussigné; toutes les citations de ce texte sont de cette traduction inédite.

2 G. Deleuze et F. Guattari, Mille plateaux, p. 296. 
pour en déceler la dimension animale appartenant proprement à l'espèce humaine.

Ainsi, le devenir animal auquel Abram initie son lecteur n'est pas une expérience d'aliénation, n'est pas un devenir autre, n'a en fait rien à voir avec la question de la différence anthropologique, celle de la singularité des humains par rapport aux autres vivants. L'enjeu concerne l'intensification et l'approfondissement de la capacité de sentir propre aux humains, capacité qui leur a permis durant des millénaires d'entretenir des relations équilibrées avec leurs entours. Cette approche implique de toute évidence le renversement d'une habitude de pensée bien établie dans nos traditions, à savoir l'idée que la spécificité des humains serait liée à des capacités que nous aurions en propre, et dont les autres animaux seraient privés. Il faut se départir de cette question pour bien saisir le propos d'Abram, et ne pas se contenter (si j'ose dire) d'une inversion de la thèse à l'instar de l'anthropologie privative élaborée par Renaud Barbaras. Cette position est une réponse puissante à la thèse selon laquelle les humains auraient quelque chose en plus par rapport aux autres animaux (le langage, la religion, le rire, le sens du réel, etc.) $)^{3}$. Selon Barbaras, les humains ont plutôt quelque chose en moins, à savoir l'expérience de la perte, qui est nécessaire pour laisser place au désir. Cependant, cette thèse est basée également sur la prémisse que la différence anthropologique se baserait sur la possession exclusive d'une capacité, ici la capacité à éprouver du désir; cette position est très proche de celle de Henri Maldiney qui soutient en plusieurs endroits que la spécificité des humains est de pouvoir faire l'expérience du vide ${ }^{4}$, qu’à la différence des animaux qui ne font que subir la vie, les humains ont une capacité de résistance qui les ouvre à l'existence proprement dite, mais qui consiste réellement en l'expérience du néant, donc à une déficience, à un manque. Sans entrer dans le détail d'une argumentation complexe, je veux seulement signaler que ces positions de Barbaras et de Maldiney reviennent au fond à inverser la thèse de l'exception humaine, et de cette manière à conférer aux humains une qualité particulière et les distinguer de cette manière des autres vivants.

3 Voir notamment R. Barbaras, Métaphysique du sentiment, p. 119-140.

4 Cf. H. Maldiney, Penser l'homme et la folie, en particulier p. 280 sq. Voir aussi le texte sur "Le vide dans la peinture occidentale», Ouvrir le rien, l'art nu, p. 111-124. 
Ce détour se justifie pour souligner que l'enjeu chez Abram est en contraste avec cette approche anthropocentrée; Abram invite à penser la particularité humaine en fonction de la manière d'exercer ses capacités, en multipliant les comparaisons avec la manière dont les autres animaux exercent les leurs, et sans jamais affirmer que l'humanité de l'homme reposerait sur une capacité qu'il est seul à posséder, ou à ne pas posséder. Il y a des manières humaines de communiquer, de se mouvoir, de percevoir, etc., et toutes trouvent leur analogue chez d'autres vivants non humains. L'enjeu est de faire l'expérience de l'animalité humaine en dialogue et en résonance avec l'animalité des autres vivants, et non pas par différence avec eux. Cette expérience est la condition de possibilité d'un lien intime avec le monde, une relation de résonance qui atteste notre appartenance aux éléments du monde.

\section{La profondeur et le corps en mouvement}

C'est le mouvement corporel et le rapport mouvant à l'espace qui constitue la dimension fondamentale de notre sensibilités. En premier lieu, notre animalité humaine est d'abord manifestée par notre corporéité et notre existence dans l'espace. Abram offre à plusieurs reprises dans le livre des descriptions phénoménologiques saisissantes des interactions du corps en mouvement et de l'espace. Dans le premier chapitre, intitulé «Ombre» et sous-titré «Écologie profonde I», il décrit comment le mouvement du soleil et des ombres changent l'expérience de l'espace sensible. Le chapitre commence par une description saisissante de l'expérience du coucher du soleil dans une vallée de montagne du Nouveau Mexique, et de la manière dont l'envahissement progressif de l'ombre dans la vallée après la disparition du soleil derrière la crête de

5 À propos de ce terme de «sensibilité», il faut faire une remarque sémantique qui vaudra pour l'entier du texte et qui permettra une lecture sans obstacle du texte d'Abram: la langue anglaise est riche de plusieurs termes signifiant la sensibilité, en particulier sensibility, sensitivity, et sentience. Le premier équivaut exactement au français sensibilité, dans le sens d'une capacité de perception et d'attention; le deuxième équivaut à la sensibilité au sens de la tendance à se laisser émouvoir, voire à la susceptibilité; le troisième, beaucoup employé par Abram, est traduit par "capacité de sentir» et désigne la faculté des êtres d'éprouver des sensations. 
montagnes modifie la texture même de l'air et des odeurs, et donc aussi le rapport du corps à lui-même. Voici un extrait de ce passage:

"C'est plus frais maintenant, assurément. Mais pas seulement plus frais - l'air a une nouvelle texture, humide: l'eau suspendue dans le milieu aérien, invu, bien qu'on sente sa présence quand les vagues de l'air lèchent ton visage. Et chevaucher ces vagues est d'abord attirant, puis irrésistible: les odeurs! Des odeurs sombres stygiennes glissant sur le miroir troublé du lac pour se mêler à la froideur humide du sol et au parfum haut perché des aiguilles vertes, et une fragrance vaguement fermentée qui se faufile dans tes narines (la crotte récente d'une créature qui s'attarde encore dans les bois). Il y a aussi la moisissure d'un vieux tronc couché, et la senteur sombre et riante de l'eau frâiche qui se presse sur le rivage (infusée de la chimie des têtards et des truites, et du tanin des feuilles immergées), et une série d'autres parfums tantôt mêlés, tantôt distincts, tous pétillants comme du vin dans cette région de ton cerveau, auparavant bercé et endormi par le reflet soporifique des rayons solaires, mais désormais réveillé et attentif à cette magie vigoureuse, comme si ton intelligence de mammifère avait brusquement lâché l'ancre et s'était soudainement trouvée ici, debout sur ses pieds, dans ces forêts humides ${ }^{6}$.

Cette longue citation est nécessaire pour faire sentir (justement) la précision et la dimension très charnelle et concrète du style d'Abram, que j'ai tenté de rendre le mieux possible, avec ses allitérations et ses assonances. Cependant, au-delà de la qualité littéraire proprement dite, c'est la manière dont les changements dans le champ perceptif sont mis en évidence: la fraîcheur nouvelle induite par la disparition du soleil, les odeurs qui émergent avec l'humidité nouvelle de l'air, l'éveil de notre capacité perceptive produit par l'effacement des rayons de lumière agressifs. L'ombre est révélatrice; elle est un milieu de perception, une qualité de l'espace qui laisse apparaître les éléments naturels autrement, en particulier par les sens auditif et olfactif. Et le passage se conclut par cette situation :

"Tu es entré dans le pays de l'ombre. Et une présence vaste et menaçante, qui se cachait quelques instants auparavant derrière la toile de lumière, marche à présent lentement vers toi à travers l'air purifié. C’est le corps vivant de la montagne elle-même»”.

6 D. Aвram, op.cit., p. 14-15.

7 Idem, p. 15. 
La nuit apporte une altération de la présence, ce qui s'explique par le rôle joué par la lumière dans la perception de la distance; le "corps vivant (littéralement: respirant) de la montagne elle-même» enveloppe tout l'espace autour d'elle, de même que mon ombre par un jour ensoleillé fait partie de mon être dans son épaisseur et dans sa profondeur. On oublie bien trop facilement, écrit Abram, que l'ombre d'un corps humain (par exemple) n'est pas seulement une forme projetée au sol par la lumière du soleil ou d'un lampadaire, en deux dimensions. Mon ombre est un espace qui a sa profondeur et dans lequel les choses apparaissent autrement qu'à la lumière du soleil. Abram décrit peu après le passage que je viens de citer le passage d'un bourdon dans l'ombre de son corps: il voit arriver le bourdon, "comme une comète erratique et ivrogne devant le ciel asphalte", puis brusquement franchir une "frontière invisible dans l'air ${ }^{8}$ et perdre son caractère éclatant, pour enfin ressortir de cette zone d'obscurité et retrouver l'éclat du soleil. Cette expérience toute simple permet de se rendre compte que l'ombre est en trois dimensions et non pas une forme plate projetée au sol. Mais pas uniquement.

Non seulement l'ombre est une forme épaisse en trois dimensions, mais encore elle est un espace dans lequel les êtres changent de texture. L'ombre de la montagne fait partie de la montagne elle-même, et quand elle m'enveloppe à la fin de l'après-midi, «je deviens une partie de sa vie, même pour un moment». Les ombres sont des "attributs qualitatifs des corps qui les sécrètent " ${ }^{9}$, l'ombre est "un espace et un temps délimité où la montagne est libre d'étaler quelque chose d'elle-même, de se faire sentir dans toute sa franchise attirant un ensemble d'autres entités et d'éléments dans un voisinage partagé, une zone d'alliances et de réciprocités ${ }^{10}$. Cet espace mouvant, qui se déplace durant la journée et qui prend toute la place la nuit (la nuit n'étant rien d'autre que l'ombre de la Terre), appartient à l'être qui le projette et qui accueille d'autres êtres dans son sein. L'ombre d'une montagne ne donne pas seulement une profondeur visuelle, au sens où certaines choses apparaissent derrière d'autres choses. Elle donne la profondeur véritablement charnelle dans laquelle les êtres apparaissent dans toute la richesse de leurs

8 Idem, p. 16.

9 Idem, p. 21.

10 Ibidem. 
interactions et interdépendances. Notre mouvement corporel est d'abord un mouvement dans la lumière et l'expérience que nous faisons de notre corps et des autres corps implique toujours une épreuve des relations ombragées.

\section{Le paysage dansant et la prestidigitation de la terre}

Ainsi, la randonnée est l'expérience philosophique privilégiée par David Abram. Ce n'est pas la seule, il y a aussi l'observation de sa fille âgée d'un an et demi dans le livre - mais la randonnée est emblématique. Elle permet de faire l'épreuve d'un lien avec la terre à l'échelle du corps, d'éprouver le mouvement du corps lui-même et de son lien avec les éléments du paysage. Elle permet aussi de comprendre ce qu'on peut entendre par la magie: marcher à travers les vallées et les forêts est une manière privilégiée de faire l'expérience de la dimension capricieuse et imprévisible de l'espace du paysage, d'un flanc de montagne qui paraît à portée de main et qui, au détour d'un virage ou avec l'envahissement du ciel par un nuage d'orage, s'avère beaucoup plus lointain et nécessiter une journée de marche en plus. Il raconte dans le chapitre sur "La profondeur" un épisode de ses randonnées qui illustre et explicite l'analogie entre l'expérience du paysage mouvant et la prestidigitation. L'analogie repose sur l'expérience de la profondeur, quand une surface en cache une autre dans le champ perceptif. Il résume l'idée comme ceci:

«La prestidigitation repose entièrement sur la dimension de la profondeur, puisque cette dimension permet aux surfaces voisines d'en cacher d'autres, au dos d'une main relâchée de cacher une balle qu'on tient doucement dans sa paume. La même profondeur qui donne aux nuages de dissoudre les rochers permet aussi aux plis pendants d'un foulard précédemment vide de cacher un lapin patient. Or la profondeur peut aussi exercer sa magie à l'air libre. Quand tu te promènes à pieds nus sur une plage, il peut arriver que tu aperçoives à une distance pas trop lointaine, un pneu abandonné à moitié enseveli dans le sable, ses fils usés exposés au soleil et aux embruns. Par contre, si tu t'approches, il se peut que tu voies ce pneu se métamorphoser soudainement en un phoque somnolant qui se réveille et aboie, ronchon, à ton adresse, puis plonge lourdement dans la mer ${ }^{11}$.

11 Idem, p. 88. 
Ces métamorphoses ont lieu en raison du mouvement de mon corps, de son interaction constante avec le paysage. Comme le souligne Maldiney, mon expérience du paysage est une expérience d'enveloppement, "l'espace du paysage est sans coordonnées ni repères. Il a pour centre ici où je suis $»^{12}$. Ce sont les éléments du paysage qui permettent d'établir des repères, et quand ils disparaissent, ou plutôt quand ils cessent d'apparaître, la désorientation peut être complète. L'expérience du pneu qui se transforme en un phoque est plutôt amusante, mais l'histoire que raconte Abram de son séjour au Népal est plus inquiétante et étrange: il marchait dans l'Himalaya népalais, dans les contreforts de l'Ama Dablam, qui culmine à plus de 6800 mètres et dont le dôme domine la vallée qui y mène, à l'instar du Cervin qui commande la vallée de Zermatt. Il voulait arriver avant la nuit dans la maison isolée d'un chamane-guérisseur (jhankri) dont il avait entendu parler dans sa recherche de magiciens locaux ${ }^{13}$. Abram marche dans la vallée sous les auspices de la montagne, et poursuit sa progression en direction du village du guérisseur, malgré les nuages de la mousson qui commencent à masquer les sommets. Au fil de sa progression, la masse nuageuse se fait plus dense jusqu'à ce que l'Ama Dablam disparaisse définitivement dans le "miasme gazeux". Abram écrit qu'il n'était alors même plus en mesure de "sentir la présence de ce sommet impérieux" ", alors même qu'il avait marché sous son autorité durant toute la journée. Le contraste entre la présence si dominante d'une montagne et sa disparition si complète lui donne l'exemple le plus pur d'une prestidigitation réussie. Il lui fallait, en tant que prestidigitateur, reproduire le tour que les nuages de la mousson lui avaient joué, à savoir faire disparaitre un être dont la présence paraissait si imposante que sa disparition était impensable, de manière analogue à l'action du prestidigitateur: "La même profondeur, qui donne aux nuages de dissoudre les rochers, permet aussi aux plis d'un foulard précédemment vide de cacher un lapin patient $»^{15}$. Lors d'un séjour récent à Zermatt, j'ai pu également faire l'expérience de l'absence du Cervin, après avoir vécu l'émerveillement de sa présence. L'absence de

12 H. Maldiney, Ouvrir le rien, l'art nu, p. 128.

13 Abram vivait comme prestidigitateur ambulant durant ses voyages en Europe et en Asie dans sa jeunesse. Il raconte le séjour chez le guérisseur dans le chapitre "Sleight of Hand (Magic I)» (p. 201-227).

14 D. Авram, op. cit., p. 87.

15 Idem, p. 88. 
la montagne est à la mesure de sa présence imposante, et le mouvement des nuages possède un tel pouvoir de prestidigitation, rendu manifeste par la forme si imposante et impérieuse de la montagne quand le temps est clair.

La profondeur est la dimension qui permet ce type d'événements: non pas seulement le fait qu'une chose apparaisse derrière une autre, mais qu'un phénomène puisse en faire disparaître un autre. Pour cela, le mouvement corporel est nécessaire, que ce soit la randonnée à travers la vallée de l'Ama Dablam, les nuages qui enveloppent la montagne en quelques instants. Les deux sont des mouvements en interaction. Les nuages enveloppent la montagne et donnent lieu ainsi à une profondeur qu'on peut qualifier de totale puisque le phénomène d'occlusion ${ }^{16}$ est complet et ainsi annulé; la montagne n'apparaît plus derrière le nuage puisque la masse nuageuse le cache entièrement, et par une sorte d'illusion rétrospective, la montagne paraît ne jamais avoir été là. Sa disparition s'étend aussi au passé ${ }^{17}$. Cette prestidigitation du paysage est une expérience clé de l'agentivité du monde, des manières qu'a le monde de faire événement et de nous solliciter. À ce stade de la présentation, il est très tentant d'évoquer la notion de transpassibilité développée par Henri Maldiney pour caractériser la capacité du sujet à se laisser surprendre, mais plutôt qu'à une description du sujet humain dans cette situation, je voudrais consacrer l'espace qui reste à une réflexion ontologique sur l'animation du monde à la suite de David Abram.

Et pour lever tout éventuel malentendu, avant d'affronter cette question, il faut souligner qu'il ne s'agit en aucun cas de justifier une conception mystique ou religieuse, magique dans le sens d'une action causale sans contact sur les choses. L'animisme ici est un mode d'apparition et de présence du monde, et dans cette perspective, la question de l'animisme mène immédiatement à celle du fondement ontologique de l'expression des choses.

16 Pour une description précise du phénomène d'occlusion, responsable de la perception de la profondeur, $c f$. J.J. Gibson, Approche écologique de la perception visuelle, p. 298 sq. et 428 sq.

17 Cet événement de disparition de la montagne peut être mise en parallèle avec la description par Maldiney de l'événement d'apparition de la montagne dans plusieurs textes à propos du Cervin; cf. Ouvrir le rien, l'art $n u$, p. $29 s q$. et $53 s q$. 


\section{L'animation du monde et la magie des choses}

Dans le chapitre intitulé "The Speech of Things» ("La parole des choses»), Abram raconte une rencontre difficile qu'il a vécue sur la côte méridionale de l'Alaska, où il faisait une excursion solitaire en kayak. Il pagaie le long de cette côte sauvage et se retrouve à l'extrémité d'un bras de mer face à une colonie de lions de mer plutôt imposants et agressifs qui font mine de sauter dans l'eau et de le poursuivre s'il essaie de s'échapper. Il leur fait face en chantant des sons qui ressemblent à leurs aboiements, mais ils restent menaçants sur leurs rochers jusqu'à ce qu'une baleine surgisse soudainement à côté du kayak et fasse plonger les lions de mer dans l'eau, sous le choc. À ce moment, entouré d'environ quatrevingts lions de mer prêts à faire chavirer le kayak, il commence à danser en levant ses bras et en les balançant de droite à gauche et de gauche à droite. À sa grande surprise, ce geste désespéré capture l'attention des lions de mer qui eux aussi se mettent à balancer leur tête, "tous à l'unisson parfait, comme une ligne de chœur à moitié immergée ${ }^{18}$. Mais à chaque fois qu'il veut arrêter sa danse pour attraper la pagaie et s'échapper, les lions se remettent à aboyer et à le poursuivre. Il est hors de question de cesser ce balancement des bras - la seule solution est de continuer le mouvement avec un seul bras est tenter de pagayer avec l'autre. Lentement et péniblement, Abram réussit à ramer d'un seul bras et contourner la foule des lions de mer en colère pour rejoindre le large.

Cette rencontre est fondatrice pour sa conception du langage et de l'expression. La synchronisation entre les mouvements de ses bras et les têtes des lions de mer est une expérience de la communauté expressive des vivants. Abram va cependant plus loin en notant que la manière de s'exprimer de chaque vivant est formée par sa structure motrice et perceptive, qui, elle, est moulée sur les conditions naturelles. Les lions de mer ont un corps que l'évolution a bien adapté à l'élément aquatique, et les éléments leur paraissent manifestement aussi expressifs. Sinon l'énorme éclaboussure provoquée par la baleine qui surgissait des profondeurs n'aurait pas provoqué de réaction chez eux. La conclusion que tire Abram de cet épisode ouvre sur cette idée d'une expressivité potentielle de tout élément naturel. 
"Certes, nous sommes tous des mammiferes - les lions de mer, la baleine et moi - mais je restais avec le sentiment d'une communauté plus élémentaire des corps, et même d'une communication partagée aussi avec les vagues qui frémissaient sous le kayak et qui giclaient leur parole sur les rochers. Aux yeux d'un animal pleinement incarné, tout mouvement peut être un geste, et tout son peut être une voix, peut être une énonciation [utterance] du monde. Et donc, pour ma propre chair de créature, de même, tout parle ${ }^{19}$.

Aux yeux d'un animal incarné, tout mouvement peut être un geste. De même que la disparition de la montagne derrière la masse nuageuse pour le randonneur solitaire, les éclaboussures des vagues sur les rochers pour les lions de mer expriment une ambiance et invitent à une attitude ou à un geste. "Tout parle à ma chair de créature", et c'est à ce niveau fondamental d'expressivité que doit être située l'origine de notre langage humain.

\section{Ontologie de la chair et animisme}

Abram est parmi les lecteurs de Merleau-Ponty qui interprètent le concept de chair d'une manière pour ainsi dire littérale, selon laquelle le monde est fait d'une matière active qui sollicite notre capacité de sentir. Jennifer McWeeny le souligne dans un article récent à propos de l'ontologie merleau-pontienne, Abram est le premier, déjà dans son ouvrage The Spell of the Sensuous en 1996, à soutenir une interprétation explicitement animiste de la philosophie tardive de Merleau-Ponty. Cette interprétation minoritaire par rapport à la plupart des autres commentateurs trouve des arguments textuels et conceptuels solides, liés à la réponse qu'on apporte à la question de savoir si le concept merleaupontien de sujet anonyme conduit à "admettre la possibilité d'une conscience générale et par conséquent des expériences partagées ${ }^{20}$. Le travail de McWeeny est précieux entre autres parce qu'il donne une lecture précise et informée des textes merleau-pontiens. Elle souligne en particulier les passages de L'Cil et l'Esprit où Merleau-Ponty évoque

19 Idem, p. 167.

20 J.McWeeny, "The Panpsychism Question in Merleau-Ponty's Ontology», p. 125. 
l'animisme des peintres qui se sentent regardés par les choses ${ }^{21}$, mais note également d'autres passages, du Visible et l'Invisible, où MerleauPonty insiste sur la différence entre ma chair (la chair de mon corps) et la chair du monde, et sur le fait que cette dernière est muette. Mais c'est l'inspiration leibnizienne qui domine selon elle l'interprétation qu'on peut faire de la notion de chair, une conception où chaque être est dans une relation expressive avec tous les autres. Merleau-Ponty généralise la structure de l'empathie en montrant que si je suis capable de percevoir les états émotionnels d'autrui, c'est que j'en ai une certaine participation, que l'autre est en quelque façon déjà présent en moi, et c'est ainsi que le philosophe «libère la capacité de sentir des limites formelles de la conscience privée » ${ }^{22}$. Elle résume cette lecture leibnizienne comme ceci:

"Dans la monadologie charnelle, chaque étant, qu’il soit humain, végétal ou minéral, est un point de vue [perspective] et tout point de vue est un étant. Les uns sont aussi dynamiques que les autres $»^{23}$.

Et elle conclut que cette conception d'une relation expressive réciproque de ma chair et de la chair du monde est «incompatible avec la reconnaissance de deux différentes sortes de chair parce qu'une asymétrie de ce genre implique l'existence de domaines inexpressifs et formellement délimités $»^{24}$.

La tâche philosophique dans ce contexte serait de décrire en quoi consiste concrètement l'activité expressive de la chair du monde, ou en d'autres termes pourquoi la relation expressive est réversible dans certains cas (celui de la subjectivité humaine) et pas dans d'autres (celui de la matière minérale par exemple). Mais cette interprétation est propre à différentes conceptions du monde: en effet, si nos habitudes de perception, profondément marquées par la pensée de Descartes, nous portent à considérer les montagnes, les rochers, les pierres ou encore les arbres comme des êtres sans capacité de sentir, tel n'est pas le cas dans

21 Cf. M. Merleau-Ponty, L'Eil et l'Esprit, p. 32: "Ce qu'on appelle inspiration devrait être pris à la lettre: il y a vraiment inspiration et expiration dans l'Être, action et passion si peu discernables qu'on ne sait plus qui voit et qui est vu, qui peint et qui est peint».

22 J. McWeenY, op. cit., p. 132.

23 Idem, p. 133.

24 Idem, p. 134. 
la plupart des cultures indigènes ${ }^{25}$. La discussion menée par McWeeny dans son article est beaucoup plus détaillée quant aux interprétations de l'ontologie de la chair; je me suis contenté ici d'indiquer la plausibilité d'une lecture panpsychiste de cette ontologie, lecture dont David Abram est un pionnier. J'espère que les expériences concrètes qu'il relate dans ses récits et ses réflexions appuient de manière crédible cette lecture.

Une telle interprétation panpsychiste de l'ontologie du sensible donnerait le fondement métaphysique d'une conscience et d'une pratique animistes qui permettraient de libérer le sentiment d'émerveillement et d'enchantement face au monde, d'affranchir ce sentiment de toute nuance de superstition ou de délire naïf. Dans le cadre métaphysique occidental traditionnel, parler de «miracle», de "merveille» ou d' "enchantement» n'a de sens que dans une alternative glauque: soit dans le cadre d'une naïveté enfantine analogue au mythe ridicule du Père Noël, soit dans le cadre d'une mystique flottante de l'inexplicable absolu. Dans les deux cas, le phénomène est refoulé, ou même forclos.

\section{Restaurer notre indigénité}

En conclusion de ces remarques, je veux proposer aux lectrices et lecteurs de David Abram une stratégie de suspension de nos préjugés métaphysiques quelque peu différente de celle professée par la phénoménologie de fauteuil ${ }^{26}$. La suspension ici serait celle du

25 McWeeny évoque un texte du philosophe et historien amérindien Vine Deloria Jr., "American Indian Metaphysics» (Power and Place: Indian Education in America, p. 2): «La meilleure description de la métaphysique indienne est la prise de conscience que le monde, et toutes ses expériences possibles, constitue une réalité sociale, une fabrique de la vie dans laquelle chaque chose a la possibilité d'établir des relations de connaissance intime parce que tout, ultimement, est relié».

26 "Armchair Philosophy", c'est la caractérisation un peu moqueuse qu'on a pu faire de la philosophie de l'intériorité, selon laquelle il suffirait de s'asseoir dans un fauteuil confortable, fermer les yeux et penser aux expériences possibles ou réelles, les décrire et en déduire les implications. La phénoménologie de fauteuil est une pratique philosophique qui suspend notre croyance spontanée en l'existence du monde "extérieur " et se focalise sur la texture des vécus subjectifs. Avec Abram, on est sorti du fauteuil et c'est le mouvement même du corps dans le paysage qui entraîne une tout autre suspension. 
partage entre l'animé et l'inanimé. En effet, lorsqu'un sujet humain fait l'expérience d'une action exercée sur lui par le monde, par exemple une expérience de guérison ou un bouleversement subi par la rencontre avec une ouvre d'art ou un paysage, notre conviction lourdement enracinée est qu'aucune explication n'est possible - il doit s'agir d'un "miracle", et nous entendons par ce mot un événement par principe inexplicable. Cette conviction repose en définitive sur le partage ontologique de l'animé et de l'inanimé: la matière est par essence inanimée, et ne peut donc pas exercer une action. C'est d'une telle évidence que je tremble en écrivant ces lignes. Cependant, on peut décider de suspendre la croyance en ce partage, et ce faisant c'est la relation de causalité qui est mise entre parenthèses, et avec elle la conviction que seuls les êtres animés peuvent initier une chaîne causale, que les êtres inanimés ne peuvent être que les objets inertes pris dans une telle chaîne, comme les dominos qui tombent les uns sur les autres parce qu'ils ont été disposés par un être animé qui a donné ensuite la première chiquenaude! Les relations entre les événements, comme la relation entre le mouvement des nuages et la disparition de l'Ama Dablam, ne sont alors plus explicables dans cette alternative: soit une relation de causalité physique qui ferait que le nuage aurait le pouvoir de faire en sorte que la montagne n'existerait plus, soit une pure illusion de l'esprit. Au contraire, c'est le phénomène de la disparition qui est pris tel qu'il se donne à l'expérience et à la place de la causalité, c'est la notion d'expression qui s'appliquerait à la relation entre les nuages et la disparition de la montagne. Le mouvement des nuages provoque la disparition de la montagne pour un sujet qui marche dans la vallée, et cet événement exprime une certaine ambiance ou humeur (le terme anglais mood comprend les deux aspects), un changement dans le mode de présence du paysage.

Dans une perspective indigène, comprise au sens d'un point de vue hérité collectivement à partir de l'expérience d'une habitation immémoriale d'un certain territoire et de la connaissance intime des puissances du paysage et de ses autres habitants, le présupposé est inverse. C'est l'habitation de la terre qui donne le cadre de toute expérience, et dans ce cadre, tout phénomène est perçu comme une expression potentielle d'un autre. Le geste fondamental de l'Europe coloniale a été de détacher l'expérience de la terre et de la considérer comme un espace, ou une surface, indifférente, sur laquelle on pouvait construire indifféremment. 


\section{Bibliographie}

Abram David, The Spell of the Sensuous. Perception and Language in a More-Than-Human World, New York: Vintage Books, 1996; trad. fr. par I. Stengers et D. Demorcy, Comment la Terre s'est tue, Paris: La Découverte, 2014.

Aвram David, Becoming Animal. An Earthly Cosmology, New York: Pantheon Books, 2010.

Barbaras Renaud, Métaphysique du sentiment, Paris: Éditions du Cerf, 2016.

Burkhart Brian, Indigenizing Philosophy through the Land. A Trickster Methodology for Decolonizing Environmental Ethics and Indigenous Futures, East Lansing (MI): Michigan State University Press, 2019.

Deloria Vine Jr, Wildcat Daniel (eds.), Power and Place: Indian Education in America, Golden (CO): Fulcrum Resources, 2001.

Gibson J. James, Une approche écologique de la perception, Paris: Éditions Dehors, 2014.

McWeeny Jennifer, "The Panpsychism Question in Merleau-Ponty's Ontology ", in: Alloa Emmanuel, Chouraqui Frank et KaushiK Rajiv (ed.), Merleau-Ponty and Contemporary Philosophy, Albany (NY): State University of New York Press, 2019, p. 121-144.

Maldiney Henri, Penser l'homme et la folie, Grenoble: Jérôme Millon,

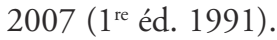

Maldiney Henri, Ouvrir le rien, l'art nu, Paris: Les Belles Lettres, 2010 ( $1^{\text {re }}$ éd. 2000).

Merleau-Ponty Maurice, L'Eil et l'Esprit, Paris: Gallimard, 1964. 